\title{
Calibration of Radars Using Polarimetric Techniques
}

\author{
Eugenio Gorgucci, Gianfranco Scarchilli and V. Chandrasekar, Member, IEEE
}

\begin{abstract}
Absolute calibration of a weather radar is critical for quantitative estimation of rainfall. This is a very difficult measurement especially for beam-filled targets like precipitation. In this paper we discuss a method that uses the properties of rain medium itself to obtain accurate system gain calibration. This technique is based on the principle that the rainfall rate measured using absolute reflectivity $(Z)$ and differential reflectivity $\left(Z_{\left[/ H_{i}\right)}\right.$ is the same as that obtained from specific differential phase $\left(K_{0}^{\prime},{ }^{\prime}\right)$. The measurements required for this technique are $Z . Z_{1, k}$, and $K_{D p}$. We compare the rainfall rate estimates $R_{l} f_{i}$ obtained from $Z$ and $\left.Z_{D}\right)$ with the estimates $R_{D P}$ obtained from $\left.K_{i}\right)$. The scatter plot between the two rainfall estimates should lie close to a 1:1 line and any systematic deviation from this line can then be removed by appropriately adjusting the system gain. It is noted here that $Z_{J) R}$ can be calibrated accurately because it is a differential power measurement and $\left.L^{\prime}\right\rangle t$, is obtained from differential phase measurement which is unaffected by system calibration. The sensitivity and accuracy of this technique are studied in this paper. We present theoretical and simulation results evaluating the accuracy of this technique for ( -band frequencies. Our analysis indicates that by sampling over few data sets of rain, the calibration can be done to an accuracy of $0.6 \mathrm{~dB}$.
\end{abstract}

\section{INTRODUCTION}

C ALIBRATION of radars is an important aspect for reliable interpretation of radar measurements. The calibration can be divided into two parts: namely, absolute and relative calibration. The relative calibration is fairly easy to obtain, where we need the transfer function of the radar system at the digital integrator output for various input levels. Even this problem could get complex if it needs to be achieved for beam-filled targets as in the case of the weather radars where the integrator output is averaged for a Rayleigh distributed signal. Gorgucci et al. [1] have developed techniques for obtaining relative calibration at different power levels using rain medium itself as a standard target. Their method is based on multiple processing of the same signal using both logarithmic and linear receivers, and using the mean statistical properties of weather echoes. This technique of relative calibration can be done fairly accurately by averaging over a large number of samples of weather signals collected with a slow scanning or stationary antenna [2]. The calibration process is complete when the relative calibration, which is the transfer function of

\footnotetext{
Manuscript received May 6, 1991: revised March 23. 1992. This work was supported by the National Group for Defense from Hydrogeological Hazards (CNR), and the USARO through the Center for Geosciences at Colorado State University.

E. Gorgucei and G. Scarchilli are with the Istituto di Fisica dell'Amosfera (CNR), Rome. Italy.

V. Chandrasekar is with the Department of Electrical Engineering, Colorado State University. Fort Collins, $\mathrm{CO} 80523$.

IEEE Log Number 9202012.
}

the radar system over different input signal levels, is coupled with an absolute reference point.

The problem of absolute calibration is more complicated for beam-filling targets as in the case of weather radars. Conventionally, absolute calibration is done by using a test sphere suspended from a balloon, receiving this signal transmitted by a standard horn or by using the sun as a standard source. The first two techniques have associated problems because they achieve point calibration and do not integrate over the radar beam completely. The solar calibration for weather radars has been used with some success since it fills $0.53^{\circ}$ in the radar beam [3]-[5]. This technique also cannot be used to perform calibration under beam-filled condition especially if the beam width is greater than $0.5^{\circ}$, which is very common. The solar flux fluctuates, and it is important to get this value cxactly for the specific measurement day. The strength of the signal received from solar flux by typical weather radars is in the order of $-100 \mathrm{dBm}$, which indicates the need for high sensitivity receivers. This paper analyzes a technique to obtain the absolute calibration of weather radars using the standard properties of rain medium. This method is similar to the principle of using rain medium as a beam-filled test target as suggested by [1]. [6], [7]. We assume C-band dual polarization system for analysis in the paper.

Our paper is organized as follows: Section II describes the self-consistency requirement of rain medium for rainfall rate comparisons based on reflectivity $(Z)$ and differential reflectivity $\left(Z_{D R}\right)$ against those based on differential phase $\left(\Phi_{D F}\right)$. Section III analyzes the extent to which this selfconsistency can be achieved due to the natural variability in rainfall. Section IV studies the limitations of this procedure due to measurement errors since $K_{D P}$ and $Z_{D R}$ could potentially have high normalized standard errors, which may affect the application of this procedure in some meteorological situations. Section $V$ summarizes the results of this paper.

\section{Dual Polarization estimation of Rainfall}

Dual polarization estimation of rainfall can be done by two methods, namely: a) based on the reflectivity measurements at horizontal $(H)$ and vertical ( $V$ ) polarizations; and b) based on differential propagation phase measurements $\Phi_{D P}$. The distribution of raindrop sizes and shapes controls the range of variability in the dual polarization estimates of rainfall. Cloud models and measurements of raindrop size distribution (RSD) at the surface and aloft show that a model of the gamma type can describe the natural variations in the RSD [8]

$$
N(D)=N_{0} D^{\mu^{\prime}}-. . D
$$




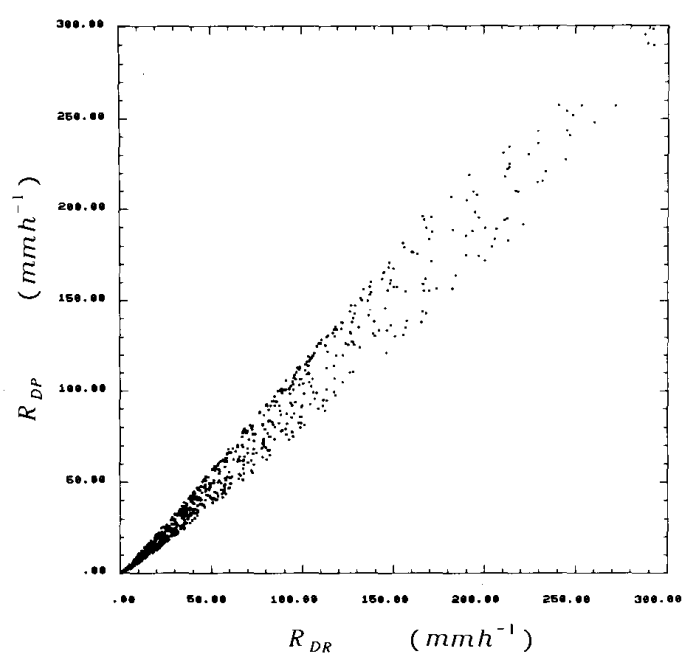

Fig. 1. Scatter plot of the rainfall rate $R_{D P}$ obtained from $K_{D P}$ versus the rainfall rate $R_{D R}$ obtained from $Z_{H}$ and $Z_{D R}$.

where $N(D)$ is the number of raindrops per unit volume per unit size interval $(D$ to $D+d D)$. The parameter $\Lambda$ is related to the drop median volume diameter $D_{0}$ as

$$
\Lambda D_{0} \approx 3.67+\mu \text {. }
$$

The nonspherical nature of raindrops describes the polarimetric response of rain medium. The equilibrium shape of a raindrop falling at its terminal fall speed is determined by the balance between the forces due to surface tension, hydrostatic pressure, and aerodynamic pressure due to airflow around the drop. The shapes of raindrops have been studied theoretically by Green [9], Beard and Chuang [10], and experimentally in wind tunnels by Pruppacher and Pitter [11] and in natural rainfall using aircraft probes by Chandrasekar et al. [12]. These studies indicate that shape of a raindrop can be approximated by an oblate spheroid with the axis ratio $(a / b)$ of the drop described by the approximate relationship

$$
\frac{b}{a}=1.03-0.062 D_{e}
$$

where $D_{e}$ is the equivolumetric spherical diameter of a raindrop in $m m$, and $a, b$ are the major and minor axes of the drop, respectively.

Reflectivity estimates are obtained from backscattered power measurements [13], whereas differential reflectivity measurements are obtained as the difference in the reflectivity between the polarizations $H$ and $V$ [14]. Mueller [15] and Sachidananda and Zrnic [16] have shown that polarized pulse pair processing technique can be used to measure the propagation differential phase shift $\left(\Phi_{D P}\right)$ which can be used to estimate the differential phase constant $\left(K_{D P}\right)$. Using theoretical analysis of dual polarization measurables in $C$-band it can be shown that the expression for rainfall estimates based on reflectivity at horizontal polarization $Z_{H}$ and $Z_{D R}$ is

$$
R_{D R}=3.61 \cdot 10^{-3} Z_{H}^{0.95} Z_{D R}^{-1.28}
$$

whereas the expression based on $K_{D P}$ is [17]

$$
R_{D P}=19.8 K_{D P}
$$

Fig. 1 shows a scatter plot of $R_{D R}$ versus $R_{D P}$ for different RSD's varying over a wide region (Ulbrich [8])

$$
\begin{gathered}
-1 . \leq \mu \leq 4 \\
10^{(3.2-\mu)} \exp (2.8 \mu) \leq N_{0} \leq 10^{(4.5-\mu)} \exp (3.57 \mu) \\
0.5 \leq D_{0} \leq 2.5
\end{gathered}
$$

We can see from Fig. 1 there is good agreement between $R_{D P}$ and $R_{D R}$ with scatter around the $45^{\circ}$ line, correlation coefficient of 0.992 and standard error of $6.5 \mathrm{mmh}^{-1}$.

\section{Absolute Gain Calibration}

The backscattered signal from weather targets received by the radar is conventionally expressed in terms of the reflectivity factor $(Z)$. The reflectivity factor is proportional to the intensity of the signal received by the radar from a resolution volume, once the transmitted power level and the radar system parameters are fixed. Since the values of the reflectivities encountered commonly in weather observations span many orders of magnitude, it is conventional to use the $\mathrm{dB}$ scale $Z(d B)=10 \log (Z)$. The $Z(d B)$ value can be related to the power received by the radar in $\mathrm{dBm}$ (power expressed in milliwatts in $\mathrm{dB}$ scale) as

$$
Z(d B)=d B m-R_{c}+20 \log R
$$

where $R$ is the range of the resolution volume under consideration and $R_{c}$ the radar constant expressed in $\mathrm{dB}$ scale. The factor $R_{c}$ is a function of several parameters of the radar system including the transmitted power, frequency, pulse width, beam width, and gain of the radar antenna. The value of $R_{c}$ is evaluated a priori and the $Z$ values are calculated from the received signal level. However, obtaining the value of $R_{c}$ accurately is a very difficult task.

Specific differential phase shift is obtained from range profile of $\Phi_{D P}$, which is unaffected by absolute calibration. $Z_{D R}$ is a differential power measurement, which can be easily corrected (for all single receiver systems) for any systematic bias by looking vertically up ( $90^{\circ}$ elevation) in rain or at very light rain situations and adjusting for zero $\mathrm{dB}$ output. Thus any bias in the estimate of $R_{c}$ translates into a bias in all the measured values of reflectivity, and any systematic bias in $Z_{H}$ will move the scatter plot (Fig. 1) away from this $45^{\circ}$ line. By correcting $Z_{H}$ (or the gain of the radar system) by an appropriate amount we can force a $45^{\circ}$ slope for the $R_{D P}$ versus $R_{D R}$ line. This procedure essentially ensures that the calibration of the radar is performed. The extent to which we can force an agreement between $R_{D R}$ and $R_{D P}$ is limited by the amount of scatter between $R_{D R}$ and $R_{D P}$.

The angular deviation of the best fit line to measured $R_{D P}$ versus $R_{D R}$ from $45^{\circ}$ is a measure of the bias. Let $\theta$ be the slope of the best fit line to $R_{D R}$ versus $R_{D P}$ data, then $\delta=\left(\theta-45^{\circ}\right)$ is a measure of the bias. Fig. 2 shows the plot of $\delta$ as a function of the bias in the system gain expressed 


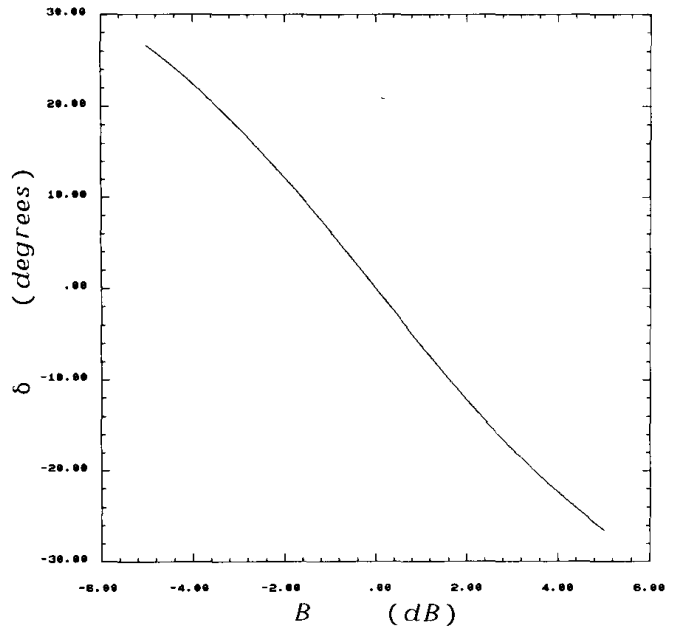

Fig. 2. Bias $\delta\left(^{\circ}\right)$ in the slope of the mean line between $R_{D P}$ and $R_{D R}$ as a function of the bias $B(\mathrm{~dB})$ in the radar system gain.

in $\mathrm{dB}$ obtained theoretically by introducing bias in the radar system gain (details of the derivation are given in Appendix I). We can see from Fig. 2 that $\delta$ is a smooth function of the bias $(B)$ and can be used to estimate $B$ from the measurement of $\theta$. Depending upon the type of rain, the scatter diagram between $R_{D R}$ and $R_{D P}$ can lie in any subset of the region of variability shown by Fig. 1 . This implies that even in the absence of any bias in the system there is no guarantee that the scatter points have to lie on both sides of $45^{\circ}$ line to obtain a mean relationship that has a slope of $45^{\circ}$, especially when we observe a limited set of rainfall types. In addition where we fit a line to a limited set of $R_{D R}$ versus $R_{D P}$ points, we need to fit a line that is forced to go through the origin, rather than a general least square fit. Thus the slope of the best fit line can be obtained as

$$
\theta=\frac{\sum_{i=1}^{N} R_{D P_{i}} R_{D R_{i}}}{\sum_{i=1}^{N}\left(R_{D P_{i}}\right)^{2}} .
$$

The estimate of $\theta$ and $\delta$ can vary depending upon the types of rainfall included in our observation. Fig. 3 shows the standard deviation of $\theta$ due to different number $M$ of rainfall types, or RSD's. We can see from Fig. 3 that data from only one type of rainfall (for example steady stratiform rain) can introduce a random error of about $3.4^{\circ}$, which translates to an error in the system gain of $0.5 \mathrm{~dB}$. If we take data over varying RSD, then the error is reduced. The results of Fig. 3 do not account for measurement errors.

\section{EfFect of Measurement Errors on Calibration}

Radar measurements of reflectivity $Z_{H}$, differential reflectivity $Z_{D R}$, and differential phase shift $\Phi_{D P}$ involve estimates of mean backscattered power from a given resolution volume at the two polarizations $H$ and $V[18]$ as well as polarizationbased pulse pair processing [16]. Fluctuations in $Z_{H}$ estimates depend on the number of samples used in the estimate as well as the Doppler spectral spread. The fluctuation in the estimates of $Z_{D R}$ and $\Phi_{D P}$ involves the cross-correlation between the

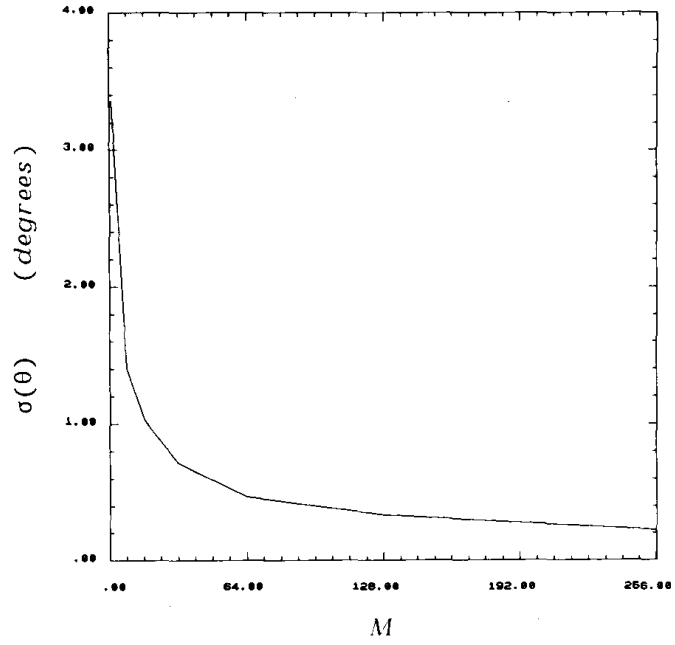

Fig. 3. Standard deviation $\sigma(\theta)\left({ }^{\circ}\right)$ in the estimate of the slope of the mean line between $R_{D P}$ and $R_{D R}$ as a function of the number $M$ of the rainfall types (or RSD's).

$H$ and $V$ signals in addition to the Doppler signal spread and the number of samples in the estimates. We use the procedure described in Chandrasekar et al. [19] to obtain estimates of $Z_{H}, Z_{D R}$, and $\Phi_{D P}$ with measurement errors. The principal parameters of the estimates are as follows: a) Wavelength: $\lambda=5.5 \mathrm{~cm}$; b) Sampling time: $T_{s}=1 \mathrm{~ms}$; c) Number of sample pairs: $N=32,64,128$; d) Doppler spectrum: Gaussian with width of $2 \mathrm{~ms}^{-1}$; e) Zero lag cross-correlation between $H$ and $V$ signals: 0.99 .

The estimates of $Z_{H}$ and $Z_{D R}$ are obtained as

$$
\begin{aligned}
Z_{H} & =\frac{1}{N} \sum_{i=1}^{N}\left(P_{H}\right)_{2 i} \\
Z_{D R} & =\frac{\sum_{i=1}^{N}\left(P_{H}\right)_{2 i}}{\sum_{i=1}^{N}\left(P_{V}\right)_{2 i+1}}
\end{aligned}
$$

where $P_{H}, P_{V}$ are the samples of backscattered power, [18]. The differential phase shift is estimated using the pulse pair algorithm modified for dual polarized signals [15], [20],

$$
\bar{\Phi}_{D P}=\frac{1}{2}\left(\psi_{1}-\psi_{2}\right)
$$

where

$$
\psi_{1}=\operatorname{Arg} \sum_{i=1}^{N} H_{2 i} V_{2 i+1}^{*}
$$

and

$$
\psi_{2}=\operatorname{Arg} \sum_{i=1}^{N} V_{2 i+1}^{*} H_{2 i+2}
$$

where $H_{2 i}$ and $V_{2 i+1}$ are the coherent complex time samples at time instants $2 i$ and $2 i+1$. Estimation of the specific differential phase $K_{D P}$ is done as follows

$$
K_{D P}=\frac{\Phi_{D P}\left(r_{2}\right)-\Phi_{D P}\left(r_{1}\right)}{2\left(r_{2}-r_{1}\right)}
$$


where $\Phi_{D P}(r)$ is the two way differential phase shift observed, at range $r$. These measurement fluctuations increase the standard errors in the estimates of $R_{D R}$ and $R_{D P}$. Fig. 4(a) shows the standard deviation $\sigma(\theta)$ in the slope estimate of the mean line between $R_{D R}$ and $R$ (true rain rate) as a function of the number $M$ of different RSD's for different number $N$ of sample pairs. We note here that $R_{D P}$ is estimated using $K_{D P}$ computed over $1 \mathrm{~km}$ path. We have chosen $1 \mathrm{~km}$ path to ensure nearly uniform rainfall path under typical rainfall conditions. For a typical 128 sample pairs situation with relatively low number of RSD's considered in the data set (for example 8 ) we can see that $\sigma(\theta)$ is in the order of 1.6 degrees. Fig. 4(b) shows similar results for $R_{D P}$ and the standard deviation of the slope estimate for the same parameters considered in Fig. 4(a) is $1.9^{\circ}$. These individual figures help us understand the contribution of different measurements to the standard deviation on observation, and could help us in the data collection process. Fig. 4(c) shows the standard deviation of the mean line slope estimate between $R_{D R}$ and $R_{D P}$ which is to be used in the calibration process. We can see from Fig. 4(c) that 128 sample pairs and relatively low number (for example 8 ) of RSD included in the data set give a standard deviation of $2.8^{\circ}$. Using the result of Fig. 2 this standard deviation translates into an error less than $0.5 \mathrm{~dB}$, which is a very little bias for calibration purpose. Fig. 5 shows the bias in $\mathrm{dB}$ versus the number $N$ of the sample pairs for different number $M$ of rainfall types. We can see that for only 8 RSD's the error is less than $1 \mathrm{~dB}$ and it reduces to $0.5 \mathrm{~dB}$ for 128 sample pairs. These observations indicate that to obtain absolute calibration to an accuracy better than $0.5 \mathrm{~dB}$, we need to sample only a few RSD's that could be obtained perhaps from monitoring only one storm through its life cycle.

\section{CONCLUSION}

The rainrate estimates $R_{D R}$ and $R_{D P}$ obtained by using $Z_{H}, Z_{D R}$, and the differential phase constant $K_{D P}$ have been compared in terms of scatter plot to determine the absolute calibration of a $C$-band weather radar. It has been shown that any systematic deviation of the slope from $45^{\circ}$ line is due to a bias on $Z_{H}$, as $Z_{D R}$ and $K_{D P}$, which are differential measurements, can be calibrated accurately. We have emphasized that this method uses the properties of rain medium itself to obtain accurate system gain calibration. It has been shown that the slope of the best fit line between $R_{D P}$ and $R_{D R}$ estimates is less sensitive to variation of rainfall rate types. Indeed, for a relatively low number of different RSD's the standard deviation of the slope estimate is $1.7^{\circ}$, which corresponds to a bias of about $0.3 \mathrm{~dB}$ on the accuracy of the system gain calibration. This accuracy can be easily obtained by sampling over few data sets of rain coming from the same storm. The measurement fluctuation on $Z_{H}, Z_{D R}$, and $K_{D P}$ increases the standard error of $R_{D P}$ and $R_{D R}$ estimates; however, it has been shown that averaging a typical number of sample pairs, i.e., 64 , the standard deviation of the slope estimate increases up to $3.8^{\circ}$ which corresponds to a bias of about $0.6 \mathrm{~dB}$. This observation indicates that to obtain absolute calibration to an accuracy better than $0.5 \mathrm{~dB}$, we

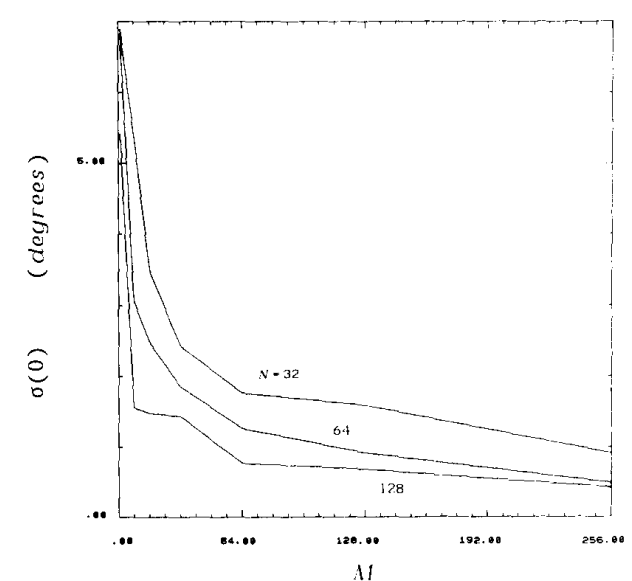

(a)

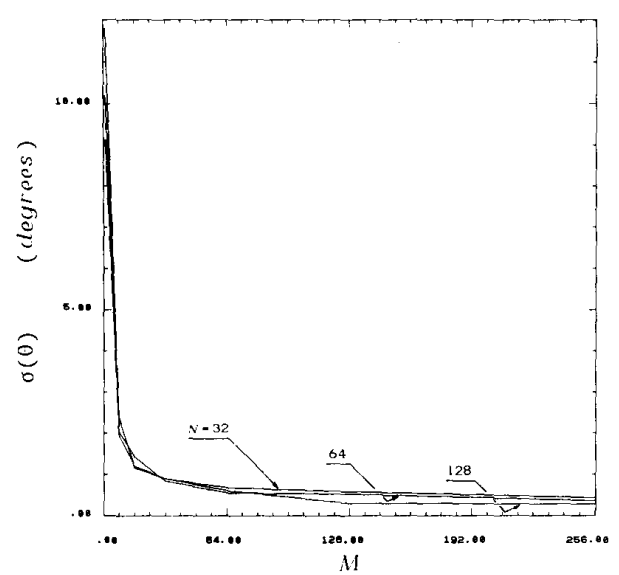

(b)

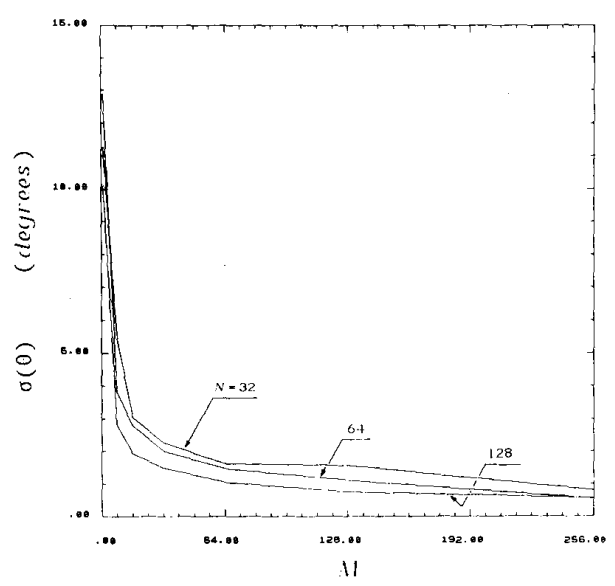

(c)

Fig. 4. (a) Standard deviation $\sigma(\theta)\left(^{\circ}\right)$ in the estimate of the slope of the mean line between the true rainfall rate $R$ and $R_{D R}$ as a function of the number $M$ of the rainfall types (or RSD's) and for different number $N$ of sample pairs. (b) Standard deviation $\sigma(\theta)\left({ }^{\circ}\right)$ in the estimate of the slope of the mean line between $R$ and $R_{D}$ pas a function of the number $M$ of the rainfall types (or RSD's) and for different number $N$ of sample pairs. (c) Standard deviation $\sigma(\theta)\left({ }^{\circ}\right)$ in the estimate of the slope of the mean line between $R_{D P}$ and $R_{D R}$ as a function of the number $M$ of the rainfall types (or RSD's) and for different number $N$ of sample pairs. 


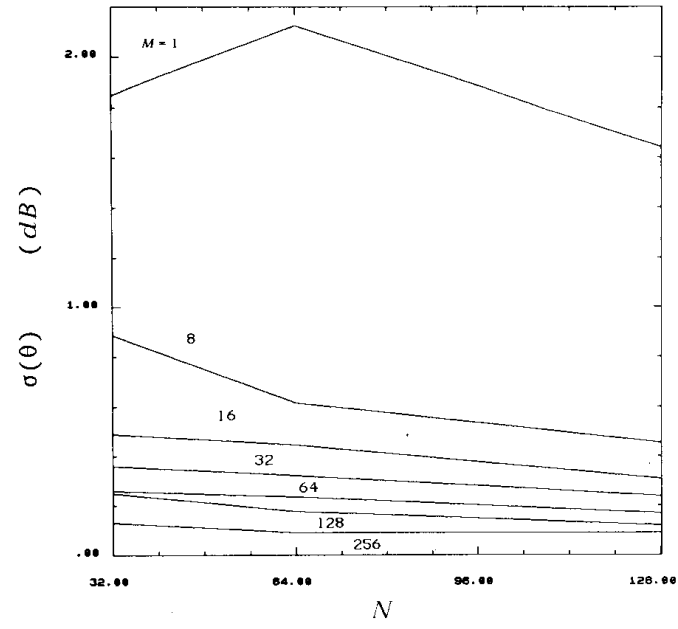

Fig. 5. Standard deviation $\sigma(\theta)(\mathrm{dB})$ in the estimate of the slope of the mean line between $R_{D P}$ and $R_{D R}$ as a function of the number $N$ of sample pairs and for different number $M$ of the rainfall types (or RSD's).

need to sample different types of rain and this could take a few days of data collection effort.

\section{APPENDIX I}

Absolute Calibration Bias as a Function of THE SLOPE OF THE SCATtERGRAM BETWEen $R_{D R}$ AND $R_{D P}$

Let $R_{D R}$ be the estimate of rainfall rate based on $Z_{H}, Z_{D R}$, and $R_{D P}$ be the estimate of rainfall based on $K_{D P}$ as described in the main text. Let $\theta$ be the angle of the position vector formed by the coordinates of $R_{D R}$ and $R_{D P}$. It follows that

$$
\tan (\theta)=\frac{R_{D P}}{R_{D R}}
$$

We can express the deviation of $\theta$ from $45^{\circ}$ as $\delta=\left(\theta-45^{\circ}\right)$. The quantities $R_{D R}, R_{D P}$, and $\delta$ are related by

$$
\tan \left(45^{\circ}+\delta\right)=\frac{R_{D P}}{R_{D R}} .
$$

In this case the estimate $R_{D R}$, taking account of (4), can be written as

$$
R_{D R}=3.61 \cdot 10^{-3}\left(\epsilon Z_{H}\right)^{0.95} Z_{D R}^{-1.28}
$$

where $\epsilon$ is the bias in the system gain. Substituting (A3) in (A1) and remembering that $R_{D P}=R_{D R}$ when no bias is present we get

$$
\tan \left(45^{\circ}+\delta\right)=\epsilon^{-0.97}
$$

The bias can be expressed in $\mathrm{dB}$ scale by taking logarithm of both sides of (A4)

$$
-0.95 \cdot 10 \log \epsilon=10 \log \tan \left(45^{\circ}+\delta\right) .
$$

This yields

$$
B(d B)=-\frac{10}{0.95} \log \tan \left(45^{\circ}+\delta\right) .
$$

Equation (A6) can be used to compute the bias in the system gain from the slope of the best fit line between $R_{D R}-R_{D P}$ scattergram.

\section{REFERENCES}

[1] E. Gorgucci, G. Scarchilli, and R. Leonardi, "Radar receiver calibration by means of multiple processing of weather echoes," J. Atmos. Oceanic Technol., vol. 3, pp. 487-499, 1989.

[2] E. Gorgucci, V. Chandrasekar, and G. Scarchilli, "Radar receiver function from correlated meteorological echoes," J. Atmos. Oceanic Techñol., vol. 8, pp. 191-197, 1991.

[3] L. J. Keeler, B. W. Lewis, and G. R. Gray, "Description of NCAR/FOF CP-2 meteorological doppler radar," preprints, 24th Conf. on Radar Meteor., Tallahassee, FL, Amer. Meteorol. Soc., pp. 589-592, 1989.

[4] C. L. Frush, "Using the sun as a calibration aid in multiple parameter meteorological radar," preprints, 22nd Conf. on Radar Meteor., Zurich, Amer. Meteor. Soc., pp. 306-311, 1984.

[5] J. F. Pratte and D. G. Ferraro, "Automated solar gain calibration," preprints 24th Conf. on Radar Meteor., Tallahassee, FL, Amer. Meteor. Soc., pp. 619-622, 1989.

[6] K. Aydin, H. Direskeneli, and T. A. Seliga, "Dual-polarization radar estimation of rainfall parameters compared with ground-based disdrometer measurements: Oct. 29, 1982 Central Illinois Experiment," IEEE Trans. Geosci. Remote Sensing, vol. 6, pp. 834-844, 1987.

[7] V. Chandrasekar, V. N. Bringi, N. Balakrishnan, and D. S. Zrnic, "Error structure of multiparameter radar and surface measurements of precipitation, Part III: Propagation differential phase shift," J. Atmos. Oceanic Technol., vol. 7, pp. 621-629, 1990

[8] C. W. Ulbrich, "Natural variations in the analytical form of raindrop size distributions," J. Climate Appl. Meteor., vol. 22, pp. 1764-1775, 1983.

[9] A. W. Green, "An approximation for the shapes of large raindrops," $J$. Appl. Meteorol., vol. 14, pp. 1578-1583, 1975.

[10] K. V. Beard and C. Chuang, "A new model for the equilibrium shape of raindrops," J. Atmos. Sci, vol. 44, pp. 1509-1524, 1987.

[11] H. R. Pruppacher and R. L. Pitter, "A semi-empirical determination of the shape of cloud and raindrops," J. Atmos. Sci., vol. 28, pp. 86-94, 1971.

[12] V. Chandrasekar, W. A. Cooper, and V. N. Bringi, "Axis ratios and oscillation of raindrops," J. Atmos. Sci., vol. 45, pp. 1325-1333, 1988.

[13] R. J. Doviak and D. S. Zrnic, Doppler Radar and Weather Observations. Orlando, FL: Academic Press, 1984

[14] T. A. Seliga and V. N. Bringi, "Potential use of the radar reflectivity at orthogonal polarizations for measuring precipitation,"J. Appl. Meteorol., vol. 15 , pp. 69-76, 1976

[15] E. A. Mueller, "Calculation procedure for differential propagation phase shift," preprints 22nd Conf. on Radar Meteor., Zurich, Amer. Meteor. Soc., pp. 397-399, 1984.

[16] M. Sachidananda and D. S. Zrnic, "Efficient processing of alternately polarized radar signals," J. Atmos. Oceanic Technol., vol. 6, pp. 173-181, 1989.

[17] G. Scarchilli and E. Gorgucci, "A correction technique for $C$-band rainfall rate estimated by means of polarimetric measurements," preprints 21st International meeting on Alpine Meteorology, Engelberg, Switzerland, pp. 57-60, 1990.

[18] V. N. Bringi. T. A. Seliga, and S. M. Cherry, "Statistical properties of the dual polarization differential reflectivity $\left(Z_{D R}\right)$ radar signal," IEEE Trans. Geosci. Remote Sensing, vol. 21, pp. 215-220, 1983.

[19] V. Chandrasekar, V. N. Bringi, and P. J. Brockwell, "Statistical properties of dual polarized radar signals," preprints 23 rd Conf. on Radar Meteor., Snowmass, Amer. Meteor. Soc., pp. 154-157, 1986.

[20] M. Sachidananda and D. S. Zrnic, "Differential propagation phase shift and rainfall rate estimation," Radio Sci., vol. 21, pp. 235-247, 1986.

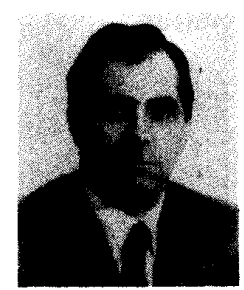

Eugenio Gorgucci received the Italian degree in physics from the University "La Sapienza" of Rome, Italy.

In 1976 he joined the Institute of Atmospheric Physics (IFA) of National Research Council (CNR) as a research scientist. His research interests are radarmeteorology, signal processing, wave propagation, and scattering. 


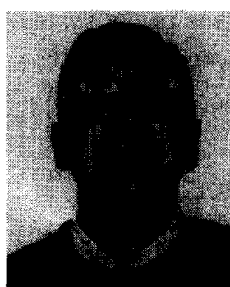

Gianfranco Scarchilli received the Italian degree in electronics engineering from the University " $\mathrm{La}$ Sapienza", Rome,Italy.

He joined the Institute of Atmospheric Physics (IFA) of the NationalResearch Council (CNR) as a research scientist. His principal research fields are radarmeteorology, signal processing, wave propagation,
V. Chandrasekar (S'83-M'83-S'84-M'87), photograph and biography not available at the time of publication. 